\title{
(2) OPEN ACCESS \\ Association of gout with CAD and effect of antigout therapy on CVD risk among gout patients
}

\author{
Wei-Shih Huang, ${ }^{1}$ Cheng-Li Lin, ${ }^{2}$ Chon-Haw Tsai, ${ }^{1}$ Kuang-Hsi Chang (D) $3,4,5$
}

${ }^{1}$ Department of Neurology, China Medical University Hospital and College of Medicine, China Medical University, Taichung, Taiwan ${ }^{2}$ Management Office for Health Data, China Medical University Hospital, Taichung, Taiwan

${ }^{3}$ Department of Medical Research, Tungs' Taichung Metroharbor Hospital, Taichung City, Taiwan ${ }^{4}$ Institute of Biomedical Sciences, China Medical University, Taichung, Taiwan ${ }^{5}$ General Education Center, Jen-Teh Junior College of Medicine, Nursing and Management, Miaoli, Taiwan

\section{Correspondence to} Dr Kuang-Hsi Chang, ---, Taichung City, Taiwan; kuanghsichang@gmail.com

C-HT and K-HC contributed equally.

Accepted 5 February 2020 Published Online First 24 February 2020
Check for updates

(C) American Federation for Medical Research 2020. Re-use permitted under CC BY-NC. No commercial re-use. Published by BMJ.

To cite: Huang $W$ S, Lin C-L, Tsai C-H, et al. J Investig Med 2020;68:972-979.

\begin{abstract}
Hyperuricemia has been identified as an independent risk factor for coronary artery disease (CAD), with a dose-response association. In this study, we explored the causal association between gout and antigout medication and the risk of incidental CAD. We sampled data from the National Health Insurance Research Database and recruited 37,091 patients as the gout cohort, and 37,091 controls. Our primary endpoint was the diagnosis of CAD during follow-up. The overall study population was followed up until CAD diagnosis, withdrawal from the National Health Insurance program, or the end of the study. Cox proportional hazards regression models were used to examine the effect of gout on the risk of CAD, represented by the HR with the $95 \%$ $\mathrm{Cl}$. Patients with gout were at greater risk of $C A D$, compared with those without gout: $H R=1.49$ after adjusting for potential confounders. Non-steroidal anti-inflammatory drugs and prednisolone use was associated with a reduced risk of $C A D: H R=0.63$ and 0.50 , respectively. Patients with gout, treated with antigout medication, exhibited a reduced risk of CAD compared with non-gout patients. Among patients with gout, those on antigout therapy had 32\% lower risk compared with those not on antigout therapy: adjusted $\mathrm{HR}=0.68,95 \% \mathrm{Cl} 0.63$ to 0.73 . Gout increases the risk of $C A D$, and the use of antigout medication reduces $C A D$ risk. These results indicate that gout or hyperuricemia is a modifiable risk factor for CAD.
\end{abstract}

\section{BACKGROUND}

The association between high levels of serum uric acid (UA) and cardiovascular disease (CVD) has long been recognized, ${ }^{1-7}$ although this has been refuted in a number of studies. ${ }^{8-11}$ Serum UA is a final enzymatic product of purine metabolism. Many studies have demonstrated that UA is a biologically active compound that can increase inflammatory mediators of vascular damage. ${ }^{2}{ }^{10-12}$ However, UA has potentially protective effects as a strong antioxidant, approaching the potency of vitamin C. ${ }^{12}{ }^{13}$ Hyperuricemia is generally defined as serum UA concentration exceeding the limit of solubility (around $6.8 \mathrm{mg} / \mathrm{dL}$ ). ${ }^{14}$ There are no universally accepted criteria to define hyperuricemia and serum UA levels ranging

\section{Significance of this study}

What is already known about this subject?

- The association between high levels of serum uric acid (UA) and cardiovascular disease (CVD) has long been recognized.

- UA is a biologically active compound that can increase inflammatory mediators of vascular damage.

- UA has potentially protective effects as a strong antioxidant, approaching the potency of vitamin C.

- Previous studies have frequently failed to distinguish whether serum UA is merely a marker for risk or a causative agent in CVD, or whether treatment targeting serum UA levels affects outcomes.

What are the new findings?

- The adjusted HR (aHR) on CVD risk was 1.49 ( $95 \% \mathrm{Cl} 1.43$ to 1.56$)$ in the gout cohort, compared with the non-gout cohort, after adjusting for age, sex, comorbidities, and medication (non-steroidal antiinflammatory drugs and prednisolone).

- Compared with controls, patients with gout who were not treated with antigout medication had a significantly increased risk of coronary artery disease (CAD) (aHR=1.62, 95\% Cl 1.55 to 1.69), followed by patients who had a history of treatment with antigout medication (aHR=1.10, $95 \% \mathrm{Cl} 1.02$ to 1.18$)$.

- Patients with gout receiving treatment exhibited a significantly lower risk of CAD (aHR $=0.68,95 \% \mathrm{Cl} 0.63$ to 0.73 ) compared with patients with gout who were not treated with antigout medication.

How might these results change the focus of research or clinical practice?

- This study supports the hypothesis that there is an increased risk of CAD among patients with gout, particularly among patients who are not treated with antigout medication, and the reduced risk in patients with gout with antigout medication treatment. Our findings indicate that gout or hyperuricemia is a modifiable risk factor for CAD. 
from 5.6 to $7.7 \mathrm{mg} / \mathrm{dL}$ in men and from 4.9 to $7.0 \mathrm{mg} / \mathrm{dL}$ in women have been used. ${ }^{1}$ Hyperuricemia and gout are closely related conditions affecting the quality of life and work productivity and are prevalent worldwide. Krishnan et al reported that hyperuricemia and untreated gout were poor prognostic markers among patients with a recent acute myocardial infarction. ${ }^{15}$ Two recent studies in 2016 also reported that higher serum UA levels were independently associated with a dose-response increased risk of coronary artery disease (CAD). ${ }^{16}{ }^{17} \mathrm{CVD}$ is one of the most common non-communicable diseases, predicted to be the major cause of morbidity and mortality in developing nations. ${ }^{18}$ The majority of CVD deaths are due to CAD. Therefore, prevention of CVD and, specifically, CAD, is clearly a major public health issue. Growing concern has mounted regarding the premature morbidity and mortality associated with CAD and there has been a recent focus on modifying risk factors and reversing the global epidemic.

Both asymptomatic hyperuricemia and gout have been identified as independent risk factors for the development and progression of CVD, CVD mortality, and all-cause mortality. ${ }^{319}{ }^{20}$ However, studies have frequently failed to distinguish whether serum UA is merely a marker for risk or a causative agent in CVD, or whether treatment targeting serum UA levels affects outcomes. In this retrospective cohort study, we postulated that hyperuricemia was a modifiable risk factor with a dose-response association with $\mathrm{CAD}$ and used a nationwide data set to determine whether gout was a predictor of CAD. We also explored the association between antigout medication treatment and CAD. We hypothesized, first, that patients with gout may have a higher risk of CAD than the general population, after adjustment for other possible demographic and metabolic confounders; and second, that antigout medication may lower CAD risk.

\section{METHODS}

\section{Data source}

In this retrospective cohort study, we used the data obtained through the Longitudinal Health Insurance Database 2000 (LHID 2000), from the National Health Insurance Research Database (NHIRD). The National Health Insurance (NHI) program was implemented in Taiwan in 1995, and provides universal coverage for most Taiwanese residents. ${ }^{21}$ The NHIRD contains data of outpatient visits, hospital admissions, prescriptions, and vital status of $99 \%$ of the 23.74 million population of Taiwan. The details of LHID 2000 have been described previously. ${ }^{22}$ Every disease diagnosis made in Taiwan is evaluated by the National Bureau of Health Insurance. Any misdiagnosis is penalized with 100-fold of the healthcare expenses reimbursed by NHI for a claimed disease diagnosis.

\section{Sampled subjects}

Patients with newly diagnosed gout (International Classification of Diseases, Ninth Revision, Clinical Modification (ICD-9-CM) code 274) were identified between January 1, 2000 and December 31, 2011; the index date was set as the day of gout diagnosis. Patients with a history of CAD (ICD-9-CM codes 410-414) before the index date were excluded. The CAD or coronary heart disease (ICD-9-CM codes 410-414) was coded when there were symptoms (chest pain as angina, or shortness of breath or heart attack) and coronary ischemic change (noted in ECG or single-photon emission CT or cardiac catheterization). The cohort without gout (ICD-9-CM code 274) was identified during the same period from the LHID 2000 using exclusion criteria comparable to the gout cohort. Each patient with gout was matched to 1 non-gout control using propensity scores. The propensity

Table 1 Characteristics between subjects with and without gout

\begin{tabular}{|c|c|c|c|c|c|}
\hline Variables & $\begin{array}{l}\text { Gout } \\
(n=37,091\end{array}$ & & $\begin{array}{l}\text { Non-gout } \\
(n=37,091)\end{array}$ & & Standard difference \\
\hline \multicolumn{6}{|l|}{ Age, y (n, \%) } \\
\hline 20-49 & 20,472 & 55.2 & 19,829 & 53.5 & 0.035 \\
\hline $50-64$ & 10,237 & 27.6 & 10,580 & 28.5 & 0.021 \\
\hline$\geq 65$ & 6382 & 17.2 & 6682 & 18.0 & 0.021 \\
\hline Age, y (mean, SD) & 48.9 & 15.4 & 49.2 & 15.6 & 0.022 \\
\hline \multicolumn{6}{|l|}{ Gender $(n, \%)$} \\
\hline Female & 8935 & 24.1 & 9211 & 24.8 & 0.017 \\
\hline Male & 28,156 & 75.9 & 27,880 & 75.2 & 0.017 \\
\hline \multicolumn{6}{|l|}{ Comorbidity (n, \%) } \\
\hline HT & 13,131 & 35.4 & 13,417 & 36.2 & 0.016 \\
\hline Diabetes & 3248 & 8.76 & 3338 & 9.00 & 0.009 \\
\hline $\mathrm{HL}$ & 11,445 & 30.9 & 11,638 & 31.4 & 0.011 \\
\hline Stroke & 865 & 2.33 & 907 & 2.45 & 0.007 \\
\hline Asthma & 1993 & 5.37 & 2045 & 5.51 & 0.006 \\
\hline COPD & 3011 & 8.12 & 3134 & 8.45 & 0.012 \\
\hline CKD & 329 & 0.89 & 325 & 0.88 & 0.001 \\
\hline \multicolumn{6}{|l|}{ Medications (n, \%) } \\
\hline NSAID & 13,021 & 35.1 & 11,743 & 31.7 & 0.07 \\
\hline Prednisolone & 3972 & 10.7 & 3951 & 10.7 & 0.002 \\
\hline
\end{tabular}

CKD, chronic kidney disease; COPD, chronic obstructive pulmonary disease; HL, hyperlipidemia; HT, hypertension; NSAID, non-steroidal anti-inflammatory drug. 


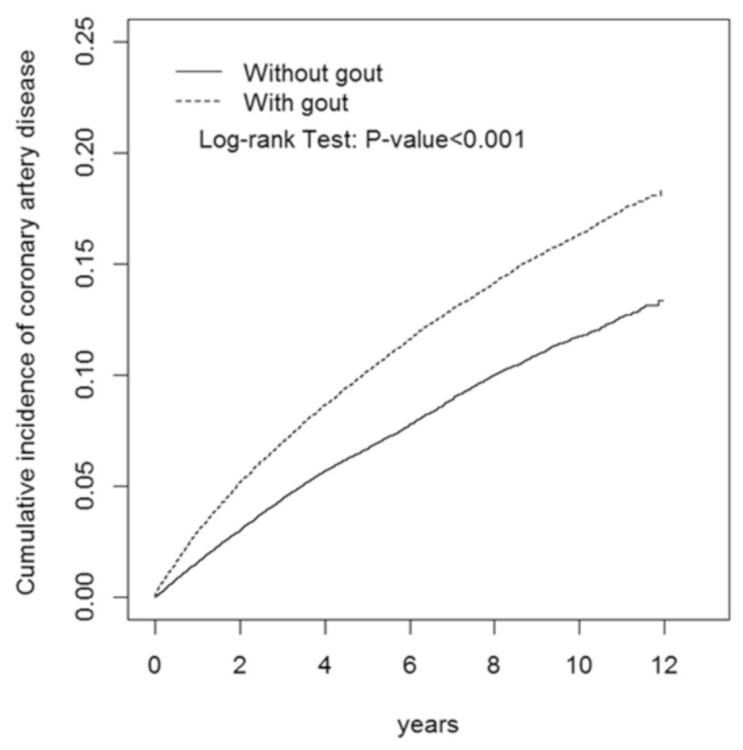

Figure 1 Cummulative incidence of coronary artery disease between subjects with and without gout.

scores were calculated by logistic regression to estimate the probability of the disease status given the baseline variables, including age, sex, year of index date, non-steroidal anti-inflammatory drugs (NSAID) and prednisolone medication, and the following comorbidities: hypertension (ICD-9-CM codes 401-405), diabetes (ICD-9-CM code 250), hyperlipidemia (ICD-9-CM code 272), stroke (ICD-9-CM codes 430-438), asthma (ICD-9-CM code 493), chronic obstructive pulmonary disease (COPD) (ICD-9-CM code 496) and chronic kidney disease (CKD) (ICD-9-CM codes 580-589). The selection of these covariates was based on the common risk factors in CAD and gout. ${ }^{24-26}$ Asthma and COPD were highly correlated with smoking consumption. ${ }^{27} 28$ Thus, we performed the multivariate analysis with age, sex and comorbidities adjustment. Both cohorts were followed up until a diagnosis of CAD was made, subjects were censored for withdrawal from the NHI program, or the date of December 31, 2011 was reached, whichever occurred first.

\section{Statistical analysis}

We compared the distribution of age, sex, comorbidities, and use of medication between the gout and non-gout cohorts using standardized differences. A value of standardized mean difference of 0.1 or less indicated a negligible difference in the mean between the gout and non-gout cohorts. The Kaplan-Meier method was used to estimate the cumulative incidence of CAD between gout and non-gout cohorts, with significance based on the log-rank test. The incidence density of CAD was estimated among different risk factors and it was stratified according to sex, age, comorbidity, and medications. Univariable and multivariable Cox proportional hazards models were performed to estimate the HR and 95\% CIs of developing CAD associated with gout. Variables in the multivariable model included age, sex, and comorbidities of hypertension, diabetes, hyperlipidemia, stroke, asthma, COPD, CKD and medication (NSAIDs and prednisolone). For further data analysis, we assessed the effects of antigout drugs (including allopurinol and benzbromarone) on the risk for CAD and compared this with the non-gout cohort. The Statistical Analysis System (SAS) V.9.4 software (SAS Institute) was used for data analysis. A two-tailed $\mathrm{p}$ value $<0.05$ was considered statistically significant.

\section{RESULTS}

We included 37,091 patients in the gout cohort and 37,091 patients in the non-gout cohort; distribution of age, sex, comorbidities, and use of medication between the 2 cohorts were comparable (table 1). Mean age was 48.9 years in the gout cohort and 49.2 years in the non-gout cohort. The majority of subjects were aged 20-49 years $(55.2 \%$ gout cohort vs $53.5 \%$ non-gout cohort), and were male $(75.9 \%$ vs $75.2 \%)$. The most prevalent comorbidity was hypertension (35.4\% vs 36.2\%) in both cohorts, followed by hyperlipidemia (30.9\% vs $31.4 \%)$, diabetes $(8.76 \%$ vs $9.00 \%)$, and COPD $(8.12 \%$ vs $8.45 \%)$. Both cohorts had a medication history of NSAIDs (35.1\% vs $31.7 \%)$ and prednisolone $(10.7 \%$ vs $10.7 \%)$. The mean follow-up period was $6.91 \pm 3.35$ years and $6.89 \pm 3.33$ years in the gout cohort and in the non-gout cohort, respectively.

The cumulative incidence curves of CAD showed that the gout cohort had a significantly higher risk of developing CAD than the non-gout cohort $(\mathrm{p}<0.001$, log-rank test) (figure 1).

Potential confounders for association of gout with risk were age, sex, comorbidities (hypertension, diabetes, hyperlipidemia, stroke, asthma, COPD, CKD), and medication (NSAIDs and prednisolone) (table 2). Compared with patients aged $\leq 49$ years old, the risk of CAD was 2.09-fold higher among patients aged 50-64 years (95\% CI 1.98 to 2.21), and 2.52-fold higher among patients aged $\geq 65$ years (95\% CI 2.36 to 2.68 ). The adjusted HR (aHR) of CAD was 1.19-fold higher among women than among men $(95 \%$ CI 1.13 to 1.25$)$. Hypertension $(\mathrm{aHR}=2.13$, $95 \%$ CI 2.02 to 2.24$)$, diabetes $(\mathrm{aHR}=1.14,95 \% \mathrm{CI}$ 1.07 to 1.22$)$, hyperlipidemia $(\mathrm{aHR}=1.41,95 \%$ CI 1.35 to 1.48$)$, COPD ( $\mathrm{aHR}=1.19,95 \%$ CI 1.11 to 1.28$)$, and CKD $(\mathrm{aHR}=1.39$, 95\% CI 1.17 to 1.65$)$ were also associated with CAD. In the multivariable model, the risk of CAD was lower among patients treated with NSAIDs $(\mathrm{aHR}=0.63$, 95\% CI 0.60 to 0.67$)$ and prednisolone $(\mathrm{aHR}=0.50,95 \% \mathrm{CI} 0.46$ to 0.54$)$.

Risk of CAD was greater in patients with gout compared with those without gout in all subgroups defined according to their demographic and clinical risk factors for CAD (figure 2, table 3).The CAD incidence was higher in women than in men, increased with age, and increased with comorbidity.

We analyzed the incidence and HR of CAD with or without antigout drug treatment (table 4). Compared with controls, patients with gout who were not treated with antigout medication had a significantly increased risk of CAD (aHR $=1.62$, 95\% CI 1.55 to 1.69$)$, followed by patients who had a history of treatment with antigout medication $(\mathrm{aHR}=1.10,95 \% \mathrm{CI} 1.02$ to 1.18$)$.

Patients with gout receiving treatment exhibited a significantly lower risk of CAD (table 5 ; aHR $=0.68,95 \%$ CI 0.63 
Table 2 The risk factors for coronary artery disease

\begin{tabular}{|c|c|c|c|c|c|}
\hline Variable & Event & PY & Rate & cHR $(95 \% \mathrm{Cl})$ & aHR $(95 \% \mathrm{Cl})$ \\
\hline \multicolumn{6}{|l|}{ Age, y } \\
\hline $20-49$ & 2456 & 300,623 & 8.17 & 1.00 & 1.00 \\
\hline $50-64$ & 3246 & 137,220 & 23.7 & 2.86 (2.71 to 3.01$)$ & 2.09 (1.98 to 2.21$)$ \\
\hline$\geq 65$ & 2501 & 73,994 & 33.8 & 4.01 (3.80 to 4.24 ) & 2.52 (2.36 to 2.68 ) \\
\hline \multicolumn{6}{|l|}{ Gender } \\
\hline Female & 2470 & 120,695 & 20.5 & 1.39 (1.32 to 1.45$)$ & 1.19 (1.13 to 1.25$)$ \\
\hline Male & 5733 & 391,143 & 14.7 & 1.00 & 1.00 \\
\hline \multicolumn{6}{|l|}{ HT } \\
\hline No & 3182 & 349,847 & 9.10 & 1.00 & 1.00 \\
\hline Yes & 5021 & 161,991 & 31.0 & 3.34 (3.19 to 3.49$)$ & $2.13(2.02$ to 2.24$)$ \\
\hline \multicolumn{6}{|l|}{ Diabetes } \\
\hline No & 7047 & 474,513 & 14.9 & 1.00 & 1.00 \\
\hline Yes & 1156 & 37,325 & 31.0 & 2.02 (1.90 to 2.15$)$ & 1.14 (1.07 to 1.22$)$ \\
\hline \multicolumn{6}{|l|}{$\mathrm{HL}$} \\
\hline No & 4430 & 359,507 & 12.3 & 1.00 & 1.00 \\
\hline Yes & 3773 & 152,330 & 24.8 & 2.00 (1.91 to 2.09 ) & 1.41 (1.35 to 1.48$)$ \\
\hline \multicolumn{6}{|l|}{ Stroke } \\
\hline No & 7963 & 502,696 & 15.8 & 1.00 & 1.00 \\
\hline Yes & 240 & 9142 & 26.3 & 1.59 (1.40 to 1.81$)$ & 0.72 (0.64 to 0.82 ) \\
\hline \multicolumn{6}{|l|}{ Asthma } \\
\hline No & 7596 & 488,154 & 15.6 & 1.00 & 1.00 \\
\hline Yes & 607 & 23,684 & 25.6 & 1.60 (1.48 to 1.74$)$ & 1.05 (0.96 to 1.15$)$ \\
\hline \multicolumn{6}{|l|}{ COPD } \\
\hline No & 7130 & 476,086 & 15.0 & 1.00 & 1.00 \\
\hline Yes & 1073 & 35,752 & 30.0 & 1.95 (1.83 to 2.08$)$ & 1.19 (1.11 to 1.28$)$ \\
\hline \multicolumn{6}{|l|}{ CKD } \\
\hline No & 8066 & 508,497 & 15.9 & 1.00 & 1.00 \\
\hline Yes & 137 & 3340 & 41.0 & 2.48 (2.10 to 2.94$)$ & 1.39 (1.17 to 1.65$)$ \\
\hline \multicolumn{6}{|l|}{ NSAID } \\
\hline No & 6197 & 303,067 & 20.5 & 1.00 & 1.00 \\
\hline Yes & 2006 & 208,771 & 9.61 & $0.49(0.47$ to 0.51$)$ & 0.63 (0.60 to 0.67$)$ \\
\hline \multicolumn{6}{|c|}{ Prednisolone } \\
\hline No & 7559 & 447,788 & 16.9 & 1.00 & 1.00 \\
\hline Yes & 644 & 64,049 & 10.1 & 0.61 (0.56 to 0.66$)$ & 0.50 (0.46 to 0.54$)$ \\
\hline
\end{tabular}

Event: $\mathrm{n}$ of coronary artery disease (CAD). Rate: incidence rate per 1000 person-years.

aHR, adjusted HR (multivariable analysis including age, gender, and comorbidities of hypertension, diabetes, hyperlipidemia, stroke, asthma, COPD, CKD, and medications of NSAID and prednisolone); CHR, crude HR; CKD, chronic kidney disease; COPD, chronic obstructive pulmonary disease; HL, hyperlipidemia; HT, hypertension; NSAID, non-steroidal anti-inflammatory drug; PY, person-years.

to 0.73 ) compared with patients with gout who were not treated with antigout medication.

\section{DISCUSSION}

We found conflicting results regarding the patients with gout who did not have higher prevalence of comorbidities except CKD in table 1 . This was most likely because the subjects without gout were significantly older than the patients with gout. The mean age was 49.2 years $(\mathrm{SD}=15.6)$ and 48.9 years $(\mathrm{SD}=15.4)$ in the non-gout cohort and gout cohort, respectively $(\mathrm{p}=0.022)$.

In this study, patients with gout had a 1.49-fold $(\mathrm{p}<0.001$, table 2$)$ higher risk of CAD than controls (representing the general population) across the 10-year study period. Moreover, compared with controls, patients with gout who were not treated with antigout medication had a higher risk of CAD, but use of antigout medication was associated with a reduced risk of CAD. These findings support our initial hypotheses and the results were statistically significant after adjustment for age, sex, and potential confounding variables, such as comorbidities. The present study demonstrates for the first time the causal relationship between gout (treated and untreated) and CAD by using a nationwide cohort data set and a follow-up study design. Analyses stratified by sex, age, and comorbidities showed that, among patients with gout, the following parameters were associated with an increased risk of CAD: female sex, the elder, lack of comorbidities, and lack of treatment with NSAIDs or prednisolone.

In this nationwide retrospective study, we evaluated the risk of CAD between the gout and control groups. Based on age, sex, medication records, and comorbidities, each patient with gout was matched to 1 control subject. However, evidence indicated that menopause increased the 


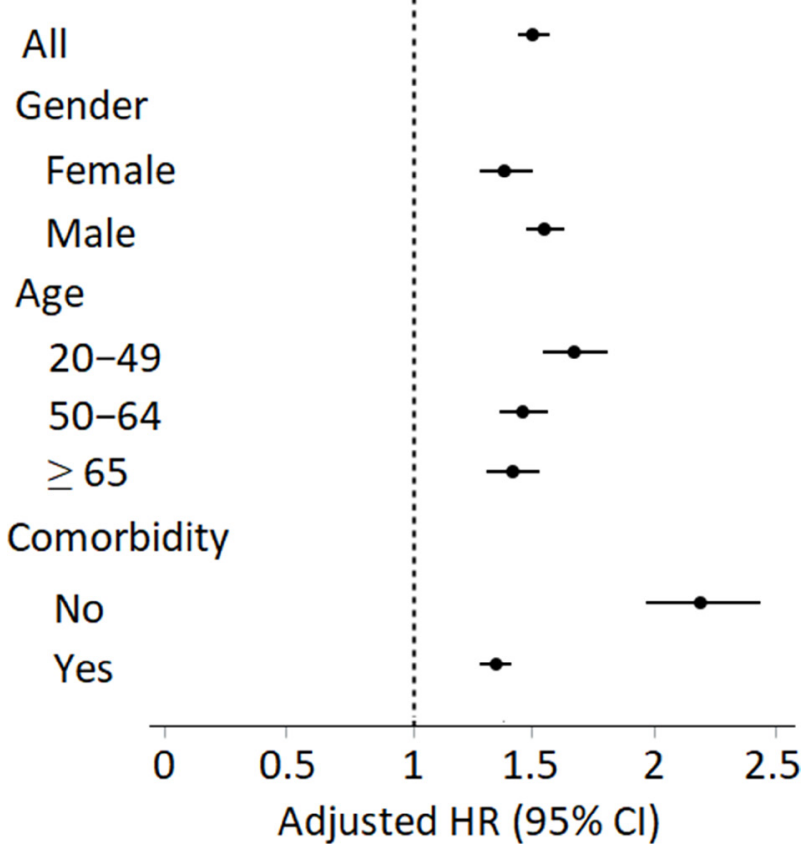

Figure 2 Adjusted HRs of coronary artery disease stratified by sex, age, and comorbidity between patients with gout compared with non-gout cohort. risk of both gout and CAD. ${ }^{29-31}$ The following conflicting finding was noted: an increased aHR for CAD in women (1.19-fold higher among women than among men, table 2).

Gout has traditionally been attributed to hyperuricemia due to accumulation of UA crystals, either due to UA overproduction or underexcretion. ${ }^{3}$ In 1886 , it was postulated for the first time, in the British Medical Journal, that serum UA may be associated with CAD. ${ }^{14}$ Subsequent studies mostly, but not invariably, supported the role of serum UA as a risk factor for CVD. This association is particularly robust among persons at high CVD risk, including those with hypertension, diabetes, CKD and congestive heart failure. $^{61617}$ A notable exception to these findings was reported in the Framingham Heart Study; here, the association between serum UA and CVD in women did not survive the adjustment for confounders and no association was observed in men. ${ }^{8}$ The prospective Chicago Industry Heart Study found serum UA levels to be independently associated with increased CVD morbidity and mortality, but only in women. ${ }^{32}$ This association found only among women was again reported in the National Health and Nutrition Epidemiologic Study 1. ${ }^{33}$ Our finding that gout was more significantly associated with CAD $(\mathrm{aHR}=1.19$, $\mathrm{p}<0.001$, table 2) in female patients than in male patients in Taiwan corroborates the 2 studies above. Our findings are also consistent with the epidemiologic characteristics

\begin{tabular}{|c|c|c|c|c|c|c|c|c|}
\hline \multirow[b]{2}{*}{ Variables } & \multicolumn{3}{|l|}{ Gout } & \multicolumn{3}{|c|}{ Non-gout } & \multirow[b]{2}{*}{ cHR $(95 \% \mathrm{CI})$} & \multirow[b]{2}{*}{ aHR $(95 \% \mathrm{Cl})$} \\
\hline & Event & PY & Rate & Event & PY & Rate & & \\
\hline \multicolumn{9}{|l|}{ Gender } \\
\hline Female & 1425 & 58,947 & 24.2 & 1045 & 61,748 & 16.9 & $1.43(1.32$ to 1.55$)$ & $1.38(1.28$ to 1.50$)$ \\
\hline Male & 3465 & 197,499 & 17.5 & 2268 & 193,644 & 11.7 & 1.50 (1.42 to 1.58$)$ & 1.55 (1.47 to 1.63$)$ \\
\hline \multicolumn{9}{|l|}{ Age, year } \\
\hline $20-49$ & 1538 & 153,712 & 10.0 & 918 & 146,911 & 6.25 & 1.60 (1.48 to 1.74$)$ & 1.67 (1.54 to 1.81$)$ \\
\hline $50-64$ & 1898 & 66,831 & 28.4 & 1348 & 70,390 & 19.2 & 1.48 (1.38 to 1.59$)$ & 1.46 (1.36 to 1.56$)$ \\
\hline$\geq 65$ & 1454 & 35,903 & 40.5 & 1047 & 38,091 & 27.5 & 1.47 (1.36 to 1.59$)$ & 1.42 (1.31 to 1.53$)$ \\
\hline \multicolumn{9}{|c|}{ Comorbidity } \\
\hline No & 1099 & 127,648 & 8.61 & 509 & 122,201 & 4.17 & 2.07 (1.86 to 2.29 ) & 2.19 (1.97 to 2.44$)$ \\
\hline Yes & 3791 & 128,797 & 29.4 & 2804 & 133,191 & 21.1 & $1.40(1.33$ to 1.47$)$ & 1.35 (1.28 to 1.41$)$ \\
\hline
\end{tabular}

Event: $\mathrm{n}$ of coronary artery disease (CAD). Rate: incidence rate per 1000 person-years. Comorbidity: subjects with any comorbidity of hypertension, diabetes, hyperlipidemia, stroke, asthma, chronic obstructive pulmonary disease (COPD), and chronic kidney disease (CKD) were classified into the comorbidity group. aHR, adjusted HR (multivariable analysis including age, gender, and comorbidities of hypertension, diabetes, hyperlipidemia, stroke, asthma, COPD, CKD, and medications of NSAID and prednisolone); cHR, crude HR; NSAID, non-steroidal anti-inflammatory drug; PY, person-years.

\begin{tabular}{lcccccc}
\hline Table 4 & Association of gout with risk of CAD subgroups defined by antigout therapy status & & \\
\hline Variable & $\mathbf{n}$ & Event & PY & Rate & cHR (95\% Cl) & aHR (95\% Cl) \\
\hline $\begin{array}{l}\text { Non-gout } \\
\text { Gout }\end{array}$ & 37,091 & 3313 & 255,392 & 13 & 1 & 1 \\
$\quad$ Without drug & 28,563 & 4014 & 195,616 & 20.5 & $1.58(1.51$ to 1.66$)$ & $1.62(1.55$ to 1.69$)$ \\
$\quad$ With drug & 8528 & 876 & 60,929 & 14.4 & $1.11(1.03$ to 1.20$)$ & $1.10(1.02$ to 1.18$)$ \\
\hline
\end{tabular}

Event: $n$ of CAD. Rate: incidence rate per 1000 person-years. Model: univariable and multivariable Cox proportional hazards models in all participants estimate the risk of developing CAD in 2 gout groups (without drug and with drug) compared with that in non-gout.

aHR, adjusted HR (multivariable analysis including age, gender, and comorbidities of hypertension, diabetes, hyperlipidemia, stroke, asthma, COPD, and medications of NSAID and prednisolone); CAD, coronary artery disease; CHR, crude HR; COPD, chronic obstructive pulmonary disease; NSAID, non-steroidal anti-inflammatory drug; $P Y$, person-years. 
Table 5 Effect of antigout therapy on risk of CAD in patients with gout

\begin{tabular}{lrrrlll}
\hline Variable & $\mathbf{n}$ & Event & PY & Rate & cHR (95\% Cl) & aHR (95\% Cl) \\
\hline Gout & & & & & & \\
$\quad$ Without drug & 28,563 & 4014 & 195,616 & 20.5 & 1 & 1 \\
$\quad$ With drug & 8528 & 876 & 60,929 & 14.4 & $0.70(0.65$ to 0.76$)$ & $0.68(0.63$ to 0.73$)$ \\
\hline
\end{tabular}

Event: $n$ of CAD. Rate: incidence rate per 1000 person-years. Model: univariable and multivariable Cox proportional hazards models in gout group estimate the risk of developing CAD in with-drug group compared with that in without-drug group.

aHR, adjusted HR (multivariable analysis including age, gender, and comorbidities of hypertension, diabetes, hyperlipidemia, stroke, asthma, COPD, and medications of NSAID and prednisolone); CAD, coronary artery disease; CHR, crude HR; COPD, chronic obstructive pulmonary disease; NSAID, non-steroidal anti-inflammatory drug; PY, person-years.

of women, who are more likely to have a higher prevalence of hypertension and high low-density lipoproteincholesterol levels than men; furthermore, menopause is also associated with a higher risk of CAD. ${ }^{3435}$

Hyperuricemia is known to be correlated with a greater risk for coronary artery calcification in young adults, which is a surrogate measure of coronary atherosclerosis. ${ }^{7}$ Two studies recently demonstrated that higher serum UA levels were independently associated with a dose-response increased risk of CAD incidence. ${ }^{16}{ }^{17}$ In the Atherosclerosis Risk in Communities Study, the OR for developing CAD was 2.59 for subjects who had a serum acid level $>9.0 \mathrm{mg} / \mathrm{dL} .{ }^{36}$ Krishnan et al reported that hyperuricemia and untreated gout were poor prognostic markers among patients with a recent acute myocardial infarction. ${ }^{15}$ The following mechanisms underlying the causal association between soluble UA and CVD have been postulated. First, UA may stimulate vascular smooth cell proliferation, reduce nitric oxide production and result in endothelial dysfunction associated with CVD. ${ }^{37}$ The endothelin-1, exerting a competent vasoconstrictive effect by binding to the receptors $\mathrm{ET}_{\mathrm{A}}$ and $\mathrm{ET}_{\mathrm{B}}$ in human vascular cells, has been postulated as a potential mechanism of an association between hyperuricemia and CVD. ${ }^{38}$ The URAT-1, a specific renal urate-anion exchange transporter as a key factor for the regulation of blood urate levels and primarily responsible for renal reabsorption of UA in the proximal convoluted tubule, is expressed in the vascular endothelial cells, as well as in the vascular smooth muscle cells. ${ }^{39}$ The presence of URAT- 1 in endothelial cells may explain the intracellular effects of urate in endothelial cells and that microvascular changes caused by hyperuricemia found in earlier rat studies, independent of hypertension or the presence of urate crystals. ${ }^{40}$ Second, inflammation is considered a key factor during the development of CAD via atherothrombosis. ${ }^{41}$ The UA has been demonstrated to be independently associated with levels of C-reactive protein (CRP), interleukin 6 (IL-6) and IL-18, and tumor necrosis factor-alpha (TNF- $\alpha){ }^{42}$ Studies in rats revealed that an artificial increase in serum UA levels results in hypertension that can be ameliorated with allopurinol. ${ }^{43} 44$ These data suggest that serum UA levels may play a role in oxidative stress and may explain why UA is associated with carotid intimal-medial thickness. ${ }^{45} 46$ Third, ischemic and dying cells release UA, which is likely to activate the immune system through dendritic cells and CD8 + T cells. Additionally, the induction of heat shock protein-70, one of the local inflammation-related proteins, can be blocked by allopurinol. ${ }^{47}$ Overall, these data suggest that UA may also play a role in the inflammatory process occurring during ischemia. Our preliminary finding that the use of antigout medication, including allopurinol and benzbromarone, in patients with gout is associated with a low risk of $\mathrm{CAD}(\mathrm{aHR}=0.68, \mathrm{p}<0.001$, table 4$)$ may support the possible role of purinergic modulators in treating CAD.

A further finding in this study is that NSAIDs, commonly used in the treatment of acute gout, decreased the risk of CAD $(\mathrm{aHR}=0.63, \mathrm{p}<0.001$, table 2$)$. The immune inflammatory reaction is involved in the pathogenesis of gout, with increased CRP levels, blood neutrophil counts, and levels of cytokines, including IL-1, IL-2, IL-6, and TNF- $\alpha{ }^{48}$ Conversely, the inflammatory reaction and associated markers are also potential etiological factors for CAD. ${ }^{40}$ NSAIDs with anti-inflammatory effects may have a role in supporting our findings.

Our study has some limitations. First, variables such as cigarette smoking, body mass index and other lifestyle factors, which may be associated with the development of CAD, were not included in our study because of the lack of data. Second, the serum UA levels are also not available in the NHIRD. Third, the population was enriched with patients with gout that may affect the risk estimates for CAD associated with gout. Finally, because all definitions of variables were based on the diagnoses made by physicians, misidentification may bias the study results. Therefore, the results should be interpreted with caution.

\section{CONCLUSION}

In conclusion, our study provides the first nationwide population-based evidence supporting the hypothesis that there is an increased risk of CAD among patients with gout, particularly among patients who are not treated with antigout medication, and the reduced risk in patients with gout with antigout medication treatment, after controlling for demographic characteristics, comorbidities, and antiinflammatory agent use. Our findings indicate that gout or hyperuricemia is a modifiable risk factor for CAD. Further prospective clinical trials are necessary to confirm this hypothesis.

Acknowledgements We thank the staff of the Management Office for Health Data of the China Medical University Hospital for their contributions to this study.

Contributors Conception/design:WSH, KHC. Provision of study materials: KHC, CLL. Collection and/or assembly of data: all authors. Data analysis and interpretation: KHC, CLL. Manuscript writing: WSH, CHT. Final approval of manuscript: all authors. 
Funding This work was supported by grants from the Ministry of Health and Welfare, Taiwan (MOHW108-TDU-B-212-133004), China Medical University Hospital, Academia Sinica Stroke Biosignature Project (BM10701010021), MOST Clinical Trial Consortium for Stroke (MOST 107-2321-B-039-004); Tungs' Taichung MetroHarbor Hospital (TTMHH-109R0010); Tseng-Lien Lin Foundation, Taichung, Taiwan, and Katsuzo and Kiyo Aoshima Memorial Funds, Japan.

\section{Competing interests None declared.}

Patient consent for publication Not required.

Ethics approval The study was approved by the Ethics Review Board of China Medical University and Hospital, Taiwan (CMUH-104-REC2-115-CR4). The Institutional Review Board waived the consent requirement.

Provenance and peer review Not commissioned; externally peer reviewed.

Data availability statement Data are available upon reasonable request. Data are available from the NHIRD published by Taiwan National Health Insurance Bureau. Due to the 'Personal Information Protection Act', data cannot be made publicly available (http://nhird.nhri.org.tw).

Open access This is an open access article distributed in accordance with the Creative Commons Attribution Non Commercial (CC BY-NC 4.0) license, which permits others to distribute, remix, adapt, build upon this work noncommercially, and license their derivative works on different terms, provided the original work is properly cited, an indication of whether changes were made, and the use is non-commercial. See: http://creativecommons.org/ licenses/by-nc/4.0/.

\section{ORCID iD}

Kuang-Hsi Chang http://orcid.org/0000-0002-4453-0068

\section{REFERENCES}

1 Alderman MH. Uric acid and cardiovascular risk. Curr Opin Pharmacol 2002;2:126-30.

2 Feig DI, Kang D-H, Johnson RJ. Uric acid and cardiovascular risk. N Engl J Med 2008:359:1811-21.

3 Gaffo AL, Edwards NL, Saag KG. Gout. hyperuricemia and cardiovascular disease: how strong is the evidence for a causal link? Arthritis Res Ther 2009; 11:240

4 Gertler MM, Garn SM, Levine SA. Serum uric acid in relation to age and physique in health and in coronary heart disease. Ann Intern Med 1951;34:1421-31.

5 Strasak AM, Kelleher CC, Brant LJ, et al. Serum uric acid is an independent predictor for all major forms of cardiovascular death in 28,613 elderly women: a prospective 21-year follow-up study. Int J Cardiol 2008;125:232-9.

6 Ito $H$, Abe M, Mifune $M$, et al. Hyperuricemia is independently associated with coronary heart disease and renal dysfunction in patients with type 2 diabetes mellitus. PLoS One 2011:6:e27817.

7 Krishnan E, Pandya BJ, Chung L, et al. Hyperuricemia and the risk for subclinical coronary atherosclerosis--data from a prospective observational cohort study. Arthritis Res Ther 2011;13:R66.

8 Culleton BF, Larson MG, Kannel WB, et al. Serum uric acid and risk for cardiovascular disease and death: the Framingham heart study. Ann Intern Med 1999;131:7-13.

9 Kavousi M, Elias-Smale S, Rutten JHW, et al. Evaluation of newer risk markers for coronary heart disease risk classification: a cohort study. Ann Intern Med 2012;156:438-44.

10 Biscaglia S, Ceconi C, Malagù $M$, et al. Uric acid and coronary artery disease: an elusive link deserving further attention. Int J Cardiol 2016;213:28-32.

11 Wu AH, Gladden JD, Ahmed M, et al. Relation of serum uric acid to cardiovascular disease. Int J Cardiol 2016;213:4-7.

12 Becker BF. Towards the physiological function of uric acid. Free Radic Biol Med 1993;14:615-31.

13 Duplancic D, Kukoc-Modun L, Modun D, et al. Simple and rapid method for the determination of uric acid-independent antioxidant capacity. Molecules 2011;16:7058-67.

14 Becker MA, Schumacher HR, Wortmann RL, et al. Febuxostat compared with allopurinol in patients with hyperuricemia and gout. $N$ Engl J Med 2005;353:2450-61. Jr.

15 Krishnan E, Pandya BJ, Lingala B, et al. Hyperuricemia and untreated gout are poor prognostic markers among those with a recent acute myocardial infarction. Arthritis Res Ther 2012;14:R10.

16 Li M, Hu X, Fan Y, et al. Hyperuricemia and the risk for coronary heart disease morbidity and mortality a systematic review and dose-response meta-analysis. Sci Rep 2016:6:19520.
17 Lai X, Yang L, Légaré $S$, et al. Dose-Response relationship between serum uric acid levels and risk of incident coronary heart disease in the Dongfeng-Tongji cohort. Int J Cardiol 2016:224:299-304.

18 Celermajer DS, Chow CK, Marijon E, et al. Cardiovascular disease in the developing world: prevalences, patterns, and the potential of early disease detection. J Am Coll Cardiol 2012;60:1207-16.

19 Baker JF, Krishnan E, Chen L, et al. Serum uric acid and cardiovascular disease: recent developments, and where do they leave us? Am J Med 2005;118:816-26.

20 Krishnan E. Gout and coronary artery disease: epidemiologic clues. Curr Rheumatol Rep 2008:10:249-55.

21 Database NHIR. Available: http://nhird.nhri.org.tw/en/index.html

22 Hsu C-L, Wang T-C, Shen T-C, et al. Risk of depression in patients with chronic rhinosinusitis: a nationwide population-based retrospective cohort study. J Affect Disord 2016;206:294-9.

23 Shih H-M, Hsu T-Y, Chen C-Y, et al. Analysis of patients with Helicobacter pylori infection and the subsequent risk of developing osteoporosis after eradication therapy: a nationwide population-based cohort study. PLoS One 2016;11:e0162645.

24 Hong J-Y, Lan T-Y, Tang G-J, et al. Gout and the risk of dementia: a nationwide population-based cohort study. Arthritis Res Ther 2015;17:139.

25 Shin JH, Kang K-W, Kim JG, et al. Concurrent renal dysfunction with ischemic heart disease is an important determinant for cardiac and cerebrovascular mortality in patients on chronic digoxin therapy for atrial fibrillation. Kidney Res Clin Pract 2018;37:130-7.

26 Wang J, Sim AS, Wang XL, et al. Relations between markers of renal function, coronary risk factors and the occurrence and severity of coronary artery disease. Atherosclerosis 2008;197:853-9.

27 Salvi S. Tobacco smoking and environmental risk factors for chronic obstructive pulmonary disease. Clin Chest Med 2014;35:17-27.

28 Santos UdeP. Asthma and smoking: still a prevailing topic. J Bras Pneumol $2016 ; 42: 81$

29 Hak AE, Curhan GC, Grodstein F, et al. Menopause, postmenopausal hormone use and risk of incident gout. Ann Rheum Dis 2010;69:1305-9.

30 Castelli WP. Epidemiology of coronary heart disease: the Framingham study. Am J Med 1984;76:4-12.

31 Maas AHEM, van der Schouw YT, Regitz-Zagrosek V, et al. Red alert for women's heart: the urgent need for more research and knowledge on cardiovascular disease in women: proceedings of the workshop held in Brussels on gender differences in cardiovascular disease, 29 September 2010. Eur Heart J 2011;32:1362-8.

32 Levine W, Dyer AR, Shekelle RB, et al. Serum uric acid and 11.5-year mortality of middle-aged women: findings of the Chicago heart association detection project in industry. J Clin Epidemiol 1989;42:257-67.

33 Freedman DS, Williamson DF, Gunter EW, et al. Relation of serum uric acid to mortality and ischemic heart disease. The NHANES I epidemiologic follow-up study. Am J Epidemiol 1995;141:637-44.

34 Lee Y, Lin RS, Sung FC, et al. Chin-Shan community cardiovascular cohort in Taiwan-baseline data and five-year follow-up morbidity and mortality. J Clin Epidemiol 2000;53:838-46.

35 Torng PL, Su TC, Sung FC, et al. Effects of menopause and obesity on lipid profiles in middle-aged Taiwanese women: the Chin-Shan community cardiovascular cohort study. Atherosclerosis 2000;153:413-21.

36 Moriarity JT, Folsom AR, Iribarren C, et al. Serum uric acid and risk of coronary heart disease: atherosclerosis risk in communities (ARIC) study. Ann Epidemiol 2000;10:136-43.

37 Waring WS, Maxwell SRJ, Webb DJ. Uric acid concentrations and the mechanisms of cardiovascular disease. Eur Heart J 2002;23:1888-9.

38 Arai $\mathrm{H}$, Hori S, Aramori l, et al. Cloning and expression of a cDNA encoding an endothelin receptor. Nature 1990;348:730-2.

39 Enomoto A, Kimura $\mathrm{H}$, Chairoungdua $\mathrm{A}$, et al. Molecular identification of a renal urate anion exchanger that regulates blood urate levels. Nature 2002:417:447-52.

40 Edwards NL. The role of hyperuricemia in vascular disorders. Curr Opin Rheumatol 2009:21:132-7.

41 Tiong AY, Brieger D. Inflammation and coronary artery disease. Am Heart J 2005:150:11-18

42 Ruggiero C, Cherubini A, Ble A, et al. Uric acid and inflammatory markers. Eur Heart J 2006:27:1174-81.

43 Johnson RJ, Kang D-H, Feig D, et al. Is there a pathogenetic role for uric acid in hypertension and cardiovascular and renal disease? Hypertension 2003;41:1183-90

44 Sánchez-Lozada LG, Tapia E, Avila-Casado C, et al. Mild hyperuricemia induces glomerular hypertension in normal rats. Am J Physiol Renal Physiol 2002:283:F1105-10. 
45 Iribarren C, Folsom AR, Eckfeldt JH, et al. Correlates of uric acid and its association with asymptomatic carotid atherosclerosis: the ARIC study. atherosclerosis risk in communities. Ann Epidemiol 1996;6:331-40.

46 Leyva F, Anker S, Swan JW, et al. Serum uric acid as an index of impaired oxidative metabolism in chronic heart failure. Eur Heart J 1997;18:858-65.
47 Shi Y, Evans JE, Rock KL. Molecular identification of a danger signal that alerts the immune system to dying cells. Nature 2003;425:516-21.

48 Cronstein BN, Sunkureddi P. Mechanistic aspects of inflammation and clinical management of inflammation in acute gouty arthritis. J Clin Rheumatol 2013;19:19-29. 